\title{
CORRECTION
}

\section{Correction to: Cellulose-based polymer electrolyte derived from waste coconut husk: residual lignin as a natural plasticizer}

\author{
Kai Ying Chua ${ }^{1}$. Ahmad Danial Azzahari ${ }^{2}$. Cheyma Naceur Abouloula ${ }^{3}$. Faridah Sonsudin ${ }^{1,4}$. \\ Nurshafiza Shahabudin ${ }^{1,4} \cdot$ Rosiyah Yahya ${ }^{2,4}$
}

Published online: 2 December 2020

(c) The Polymer Society, Taipei 2020

\section{Correction to: Journal of Polymer Research (2020) 27: 115 https://doi.org/10.1007/s10965-020-02110-8}

The original version of this article unfortunately contained many mistakes with regard to some mathematical expressions and to Tables $3 \& 5$. The correct presentations are enumerated below:

A) Equations 1-10 should be presented as:

$$
\begin{aligned}
& \mathrm{CI}(\%)=\frac{\mathrm{I}_{002}-\mathrm{I}_{\mathrm{am}}}{\mathrm{I}_{002}} \times 100 \% \\
& \mathrm{CI}(\%)=\frac{\mathrm{A}_{\mathrm{T}}-\mathrm{A}_{\mathrm{a}}}{\mathrm{A}_{\mathrm{T}}} \times 100 \% \\
& \sigma=\frac{t}{\mathrm{R}_{\mathrm{b}} \times \mathrm{A}} \\
& Z_{C P E}=k \frac{\left[\cos \left(\frac{\pi p}{2}\right)-j \sin \left(\frac{\pi p}{2}\right)\right]}{\omega^{p}}
\end{aligned}
$$

The original article can be found online at https://doi.org/10.1007/ s10965-020-02110-8

Rosiyah Yahya

rosiyah@um.edu.my

1 Centre for Foundation Studies in Science, Universiti Malaya, 50603 Kuala Lumpur, Malaysia

2 Department of Chemistry, Faculty of Science, Universiti Malaya, 50603 Kuala Lumpur, Malaysia

3 Nanomaterials for Energy and Environment Laboratory (LN2E), Faculty of Science Semlalia Marrakesh (FSSM), University of Cadi Ayyad, 40009 Marrakesh, Morocco

4 Centre for Ionics Universiti Malaya (C.I.U.M.), Faculty of Science, Universiti Malaya, 50603 Kuala Lumpur, Malaysia

$$
Z^{\prime}=\frac{R_{\mathrm{b}}+R_{\mathrm{b}}^{2} k_{1}^{-1} \omega^{p_{1}} \cos \left(\frac{\pi p_{1}}{2}\right)}{1+2 R_{b} k_{1}^{-1} \omega^{p_{1}} \cos \left(\frac{\pi p_{1}}{2}\right)+R_{\mathrm{b}}^{2} k_{1}^{-2} \omega^{2 p_{1}}}+\frac{k_{2} \cos \left(\frac{\pi p_{2}}{2}\right)}{\omega^{p_{2}}}
$$

$Z^{\prime \prime}=\frac{R_{b}^{2} k_{1}^{-1} \omega^{p_{1}} \sin \left(\frac{\pi p_{1}}{2}\right)}{1+2 R_{b} k_{1}^{-1} \omega^{p_{1}} \cos \left(\frac{\pi p_{1}}{2}\right)+R_{b}^{2} k_{1}^{-2} \omega^{2 p_{1}}}+\frac{k_{2} \sin \left(\frac{\pi p_{2}}{2}\right)}{\omega^{p_{2}}}$

$K=n e^{2} a^{2} \gamma k^{-1}$

$\omega_{p}=\frac{\sigma T}{K}$

$$
\mu=\frac{\sigma}{n e}
$$

$$
D=\frac{k T \sigma}{n e^{2}}
$$


B) Tables 3 and 5:

Table $3 \% \mathrm{CI}$ of native cellulose and $\mathrm{CMC}$

\begin{tabular}{|c|c|c|c|c|c|c|c|c|}
\hline \multirow{3}{*}{ Sample } & \multicolumn{5}{|c|}{ Peak Area } & \multicolumn{3}{|l|}{ CI (\%) } \\
\hline & \multirow[t]{2}{*}{ Peak 1} & \multirow[t]{2}{*}{ Peak 2} & \multirow[t]{2}{*}{ Peak 3} & \multirow[t]{2}{*}{ Peak 4} & \multirow[t]{2}{*}{ Peak 5} & \multicolumn{2}{|l|}{ Cellulose } & \multirow{2}{*}{$\begin{array}{l}\text { CMC (-C:30:5) * } \\
\text { Peak Height }\end{array}$} \\
\hline & & & & & & Deconvolution & Peak Height & \\
\hline CH-A-OB & 1.954 & 1.552 & 1.708 & 2.902 & 1.946 & 83.03 & 5.39 & 50.47 \\
\hline CH-A-1B & 1.028 & 1.883 & 0.625 & 2.245 & 1.712 & 91.66 & 41.53 & 38.88 \\
\hline $\mathrm{CH}-\mathrm{A}-2 \mathrm{~B}$ & 0.679 & 1.128 & 0.520 & 2.910 & 1.684 & 92.49 & 48.04 & 45.21 \\
\hline $\mathrm{CC}$ & 0.408 & 0.363 & 0.000 & 0.299 & 1.725 & 100.00 & 95.24 & 45.51 \\
\hline
\end{tabular}

*CI of carboxymethylated sample with assigned formulation -C:30:5

\begin{tabular}{lcccclcl}
\hline Samples & $\begin{array}{l}n\left(\times 10^{14}\right) \\
\left(\mathrm{cm}^{-3}\right)\end{array}$ & $\begin{array}{l}K\left(\times 10^{-16}\right) \\
\left(\mathrm{S} \mathrm{cm}^{-1} \mathrm{~K} \mathrm{~Hz}^{-1}\right)\end{array}$ & $\begin{array}{l}\omega_{p}\left(\times 10^{10}\right) \\
(\mathrm{Hz})\end{array}$ & $\begin{array}{l}\mu\left(\times 10^{-3}\right) \\
\left(\mathrm{cm}^{2} \mathrm{~V}^{-1} \mathrm{~s}^{-1}\right)\end{array}$ & $\begin{array}{l}D\left(\times 10^{-5}\right) \\
\left(\mathrm{cm}^{2} \mathrm{~s}^{-1}\right)\end{array}$ & $\begin{array}{l}\sigma\left(\times 10^{-4}\right) \\
\left(\mathrm{mS} \mathrm{cm}^{-1}\right)\end{array}$ \\
\hline CH-A-1B- & C:30:5 & 2.55 & 4.27 & 9.77 & 3.37 & 8.79 & 1.38 \\
& C:30:6 & 14.6 & 24.4 & 3.10 & 1.07 & 2.79 & 2.50 \\
& C:38:5 & 4.95 & 8.29 & 17.6 & 6.07 & 15.8 & 4.82 \\
CH-A-2B- & C:30:5 & 7.33 & 12.3 & 1.19 & 0.41 & 1.07 & 0.48 \\
& C:30:6 & 5.88 & 9.84 & 4.17 & 1.44 & 3.75 & 1.35 \\
& C:38:5 & 1.04 & 1.74 & 6.49 & 2.24 & 5.84 & 0.37 \\
\hline
\end{tabular}

Table 5 Transport property parameters of CMC- $\mathrm{NH}_{4} \mathrm{NO}_{3}$ $\mathrm{PE}$ system at ambient temperature

Publisher's note Springer Nature remains neutral with regard to jurisdictional claims in published maps and institutional affiliations. 